\title{
Anti-PD1 monotherapy in hepatocellular carcinoma: a step forward or already behind?
}

\author{
Silvia Catanese $e^{1,2}$, Florian Lordick ${ }^{2}$ \\ ${ }^{1}$ Department of Medical Oncology, Azienda Ospedaliero Universitaria Pisana (AOUP), University of Pisa, Pisa, Italy; ${ }^{2}$ Department of Medicine-II \\ (Oncology, Gastroenterology, Hepatology, Pulmonology, and Infectious Diseases), University Cancer Center Leipzig (UCCL), Leipzig University \\ Medical Center, Leipzig, Germany \\ Correspondence to: Prof. Dr. Florian Lordick. Department of Medicine-II (Oncology, Gastroenterology, Hepatology, Pneumology, Infectiology), \\ Leipzig University Medical Center, Liebigstreet 22, 04103 Leipzig, Germany. Email: direktion.uccl@medizin.uni-leipzig.de. \\ Comment on: Qin S, Ren Z, Meng Z, et al. Camrelizumab in patients with previously treated advanced hepatocellular carcinoma: a multicentre, open- \\ label, parallel-group, randomised, phase 2 trial. Lancet Oncol 2020;21:571-80.
}

Submitted Jun 02, 2020. Accepted for publication Jun 12, 2020.

doi: $10.21037 /$ atm-20-4438

View this article at: http://dx.doi.org/10.21037/atm-20-4438

In The Lancet Oncology, Qin et al., presented the promising results of a multicentre, open-label, parallel-group, randomised, phase 2 trial in a Chinese population, exploring the efficacy and safety of two different application schedules of the anti-PD-1 monoclonal antibody Camrelizumab as post-progression treatment of advanced hepatocellular carcinoma (1). Eligible patients were either refractory or intolerant to first line systemic therapy. Different from other studies on PD-1 inhibitors in HCC, HBV infection was identified in more than $80 \%$ of cases, which reflects the typical HCC epidemiology in China. Noteworthy is the high prevalence of other baseline characteristics which usually indicate a poor prognosis: $95 \%$ of patients had Barcelona Clinic Liver Cancer (BCLC) stage C HCC, $12 \%$ had macrovascular invasion, $82 \%$ showed extrahepatic spread and $>50 \%$ an alpha-fetoprotein (AFP) blood level of $\geq 400 \mathrm{ng} / \mathrm{mL}$. After a median follow-up of 12.5 months [inter-quartile range (IQR) 5.7-15.5], the objective response rate (ORR; primary endpoint) and the 6-month overall survival were $14.7 \%$ (95\% CI: $10.3-20.2 \%$ ) and $74.4 \%$ (95\% CI: 68.0-79.7\%), respectively. Median overall survival (OS) and progression free survival (PFS) were 13.8 months (95\% CI: 11.5-16.6) and 2.1 months (95\% CI: 2.0-3.2), respectively. However, OS analysis is still immature with only $53 \%$ of patients who had died at cut-off date. Interestingly, of the 161 patients who had initial radiological disease progression per investigator, 95 (59\%) continued camrelizumab treatment (monotherapy or in combination with, or followed by, other therapy). Subsequent treatments received by the 66 patients who discontinued treatment with the immune checkpoint inhibitor included any kind of molecularly targeted therapy in $41 \%$. Patients who continued camrelizumab had an overall survival probability at 6 months of $74 \%$ compared to only $54.4 \%$ in those patients who discontinued camrelizumab. The most common treatment-emergent immune-related adverse event was reactive cutaneous capillary endothelial proliferation (RCCEP) (67\% of grade 1-2, none of grade 3) which represents a drug-specific, early onset cutaneous side-effect, apparently associated with treatment response on post-hoc analyses (1). Although further investigations are needed, the same association with response was recently observed in a phase I study with camrelizumab (2) in a variety of solid tumours and in a post-hoc exploratory analysis from the phase III ESCORT trial investigating camrelizumab vs. investigator's choice as second line treatment in squamous cell esophageal carcinoma (3). Other toxicities were as expected from this class of drugs. Since the primary endpoint analysis of ORR is promising and the safety profile is manageable, camrelizumab might be considered as a potential postprogression treatment option for advanced HCC, especially 
in a population with poor prognostic characteristics, according to authors' opinion (1). In our opinion, this statement deserves some discussion in the rapidly advancing field of immune-therapy for HCC.

HCC is the most common type of primary liver cancer. The target organ of HCC is a central player of immunomodulation. It ensures protection of the organisms by removing continuously a large spectrum of pathogens, microbe-associated molecular patterns (MAMPs) and damage associated molecules (DAMPs) while maintaining immune-tolerance to non-pathological or constant inflammatory stimuli. Deregulation of this tightly controlled immunological network can lead to liver disease, including liver cirrhosis and liver cancer. The multitude of the hepatic resident innate and adaptive immune cells (Kupffer cells, dendritic cells, liver sinusoidal endothelial cells, NK T cells, $\gamma \delta \mathrm{T}$ cells, Ito cells, CD8+ and Cd4+ $\mathrm{T}$ cells) and their swinging phenotype between immuneactivation and immune-tolerance constitute the background for the ongoing paradigm shift towards immunotherapy in HCC (4). Camrelizumab is a high affinity monoclonal antibody directed against the negative immune-regulatory human cell surface receptor programmed death-1 (PD-1). By blocking the binding of PD-1 on activated T lymphocytes, B and natural killer (NK) cells to its ligands programmed cell death ligand 1 (PD-L1), which can be found to be overexpressed on cancer cells, and programmed cell death ligand 2 (PD-L2), primarily expressed on antigen presenting cells (APCs), it restores immune function against tumor cells or pathogens. Compared to the PD-1 inhibitors, nivolumab and pembrolizumab, camrelizumab shows persistent receptor occupancy and binds to a different epitope leading to subtle differences in signaling regulation. Of note, it also shows a potent agonism on vascular endothelial growth factor receptor 2 (VEGFR2) being most probably responsible for its highly specific vascular neogenesis side effect (hemangioma proliferation). However, the fact that it affects preferably the skin and not mucosal tissues, downsizes the risk for bleeding and for critical drug tolerability (5-7). The paramount question, nevertheless, is if we need another mono-immunotherapy agent as second line treatment: does camrelizumab represent a novel cutting-edge strategy?

Although formal comparisons among different studies should not be done, both pembrolizumab and nivolumab demonstrated interesting activity in second line treatment of advanced HCC (8-10). The ORR with pembrolizumab in the phase 2 Keynote-224 (17\%, 95\% CI: 11-26\%) and in the corresponding phase 3 Keynote-240 (18.3\%, 95\% CI: $14-23.4 \%$ ) tended to be higher than the ORR reported with camrelizumab. Of note, more favorable baseline characteristics of patients included in the Keynote studies may explain these differences. $(8,9)$ With an ORR of $13 \%$ in the chronic hepatitis related advanced HCC subgroup of Keynote-224 (45\% of the overall population) no difference in terms of anti-tumor activity seems to exist between pembrolizumab and camrelizumab for this subgroup of patients (8). In Keynote-240, pembrolizumab unfortunately did not reach its predetermined level of statistical significance for an improvement in survival, although at the two exploratory sensitivity analyses, which evaluated OS adjusting for the use of subsequent treatment, the HR for OS was 0.65. An explanation is that the control arm performed better than expected, probably due to the increasing use of effective post-progression therapies (9,11-13). In a phase $1 / 2$ study, also nivolumab showed noteworthy and durable responses both in treatment-naïve patients and in patients progressing on sorafenib (ORR $21-23 \%$; 9-month survival rate of $74 \%$ ). Especially, the duration of response was impressive. Albeit the study was not powered for statistical comparison between patients with different HCC etiologies including viral hepatitis, responses seemed to be consistent among the different HCC groups. In the subgroup of $51 \mathrm{HBV}$ positive patients presenting with extrahepatic spread, macrovascular invasion and high levels of $\alpha$-fetoprotein, that were comparable with those of the Chinese population treated with camrelizumab, an ORR of $14 \%$ and a 6 months OS of $84 \%$ were observed, which are in line with the efficacy of all other anti-PD1 inhibitors (10). Nevertheless, the randomized controlled phase III Checkmate-459 study, comparing nivolumab with sorafenib in first-line advanced HCC, also did not reach the predefined threshold of statistical significance for better OS. A relevant difference in terms of higher response rates with nivolumab did not translate into better OS (median 16.4 vs. 14.7 months); nonetheless, like in the Keynote-240, the control arm performed better as expected, probably due to the subsequent use of systemic therapies, including immunotherapy (14). Therefore, we might conclude that the OS benefit achieved by immunecheckpoint monotherapy is meaningful, even more when considering the better quality of life reported by patients in the immunotherapy arms. It should be considered, though, that $30-40 \%$ of patients did not respond at all to immunotherapy, and disease progressed quickly in some cases. Therefore, the lack of good predictive markers is 
one of the major limitations for the successful use of PD-1 inhibitors as monotherapy, but the fundamental one is the forthcoming amendment of our therapeutic strategy due to the results of the Imbrave-150 trial (15).

The Vascular endothelial growth factor-A (VEGF-A) is-for more than a decade-a major therapeutic target in HCC first line therapy (16-18). Moreover, it is well known that tumor angiogenesis and immunosuppression in cancer pathogenesis are connected: tumor dissemination requires neo-vasculature and suppression of excessive inflammation (19). Tumor cells and endothelial cells release abundant amounts of VEGF-A, which activates and recruits the immunesuppressive cell department including tumor-associated macrophages, regulatory T-cells (T-REGs) and myeloidderived suppressor cells (MDSC). This contribute to the immune-escape mechanisms of HCC. Thus, the disruption of angiogenesis, which has become a cornerstone of HCC treatment, theoretically enhances the efficacy of immunebased cancer therapies, the new avant-garde of the HCC therapeutic scenario. Experimental evidence shows an improvement of antigen presentation by dendritic cells, an increase of T-cell priming and inhibition of T-REGs by reducing the release of inhibitor cytokines, like TGF- $\beta$ and IL-10, and eventually a regulation of the T-lymphocyte traffic from lymph nodes to tumor site $(20,21)$. Clinically, the combination of anti-angiogenics and immunecheckpoint inhibitors in HCC has revealed outstanding results (22-24). Recently, atezolizumab, a PD-L1 targeting monoclonal antibody, and bevacizumab, a VEGF-A directed agent, have demonstrated superiority in terms of ORR and OS in treatment-naïve advanced HCC patients compared with standard of care. A notable ORR of $27.3 \%$ vs. $11.9 \%$ translated into a better PFS (6.8 vs. 4.3), a considerable duration of response, which was longer than 6 months in $87.6 \%$. The median OS was not reached in the experimental arm vs. 13.2 months in the control arm (HR 0.58, 95\% CI: $0.42-0.79 ; \mathrm{p}<0.001$ ) with a rapid achievement of the plateau in the atezolizumab plus bevacizumab group; estimated rates of survival at 6 and 12 months were $84.8 \%$ and $67.2 \%$ vs. $72.2 \%$ and $54.6 \%$. The safety of immune-checkpoint inhibition and anti-angiogenesis was adequate and in terms of number of reported events comparable with sorafenib. The trial is even more noteworthy as the study population shows high-risk features: $38 \%$ of macro-vascular invasion, including invasion of the major portal trunk, which is often an exclusion criterion in advanced HCC first line trials; $38 \%$ of patients showed alpha-fetoprotein higher than $400 \mathrm{ng} / \mathrm{mL} ; 49 \%$ had an underlying chronic HBV infection, $63 \%$ showed extrahepatic spread of disease. The magnitude of the effect in co-primary endpoints in the Chinese population, characterized by higher proportion of the abovementioned risk factors (88\% HBV positive and $86 \%$ in BCLC-C stage), was clinically meaningful and consistent with that of the global population (HR for OS: $0.44 v s .0 .58$ and for PFS: 0.6 vs. 0.59) (15). The early separation of the OS curves, maintained over the time, has to be highlighted as well, despite a higher proportion of patients in the sorafenib group receiving subsequent treatment, including immunotherapy.

We might also speculate about the mechanism of action of camrelizumab: its agonism on VEGFR2 may contradict the strong rationale and the results derived from the combination of antiangiogenic therapies and immunecheckpoint inhibitors previously shown. In line with these considerations, the combination of camrelizumab with apatinib (anti-VEGFR2) in a phase $1 \mathrm{~b}$ trial that included 43 patients affected by HCC and gastroesophageal cancers obtained an ORR of $30.8 \%$ (95\% CI: $17.0-47.6 \%$ ); moreover, in $50 \%$ of HCC patients a partial response was observed (24). A trial aiming to validate these results in the first line setting is ongoing (see Table 1).

In summary, the perspectives derived from Imbrave- 150 in the first line setting and the awaited results from ongoing studies of combination therapies are expected to change the HCC therapeutic algorithms profoundly. The cruising speed of this motion is so high that goals like those achieved by anti-PD 1 monotherapy with camrelizumab after progression to sorafenib, seem to be already out of date. 
Table 1 Selected ongoing trials in HCC treatment

\begin{tabular}{|c|c|c|c|c|c|c|}
\hline $\begin{array}{l}\text { Clinical trial } \\
\text { identifier }\end{array}$ & Setting & Phase & Treatment arm & $\begin{array}{l}\text { Primary } \\
\text { endpoint }\end{array}$ & Recruiting & $\begin{array}{c}\text { Target } \\
\text { accrual } \\
\text { (pts) }\end{array}$ \\
\hline NCT03298451 Himalaya & First line & III & Durvalumab + Tremelimumab vs. Sorafenib & OS & No & 1,310 \\
\hline NCT03713593 LEAP-002 & First line & III & Lenvatinib + Pembrolizumab vs. Lenvatinib & PFS, OS & No & 750 \\
\hline NCT04039607 CheckMate 9DW & First line & III & Nivolumab + Ipilimumab vs. Sorafenib or Lenvatinib & os & Yes & 1,084 \\
\hline NCT03412773 Rationale-301 & First line & III & Tislelizumab vs. Sorafenib & OS & No & 674 \\
\hline NCT03778957 Emerald-1 & $\begin{array}{l}\text { First line in } \\
\text { aLLD }\end{array}$ & III & TACE Durvalumab +/- Bevacizumab vs. TACE & PFS & Yes & 600 \\
\hline NCT03383458 Checkmate-9Dx & Adjuvant & III & Nivolumab vs. Placebo & RFS & Yes & 530 \\
\hline NCT03867084 Keynote-937 & Adjuvant & III & Pembrolizumab vs. Placebo & RFS, OS & Yes & 950 \\
\hline NCT03847428 Emerald-2 & Adjuvant & III & Durvalumab +/- Bevacizumab vs. Placebo & RFS & Yes & 888 \\
\hline NCT04102098 Imbrave-050 & Adjuvant & III & Atezolizumab+ Bevacizumab vs. Placebo & os & Yes & 662 \\
\hline
\end{tabular}

pts, patients; aLLD, advanced liver limited disease; ORR, objective response rate; PFS, progression free survival; RFS, relapse free survival; OS, overall survival; NCT, Number of Clinical Trial (https://clinicaltrials.gov/).

\section{Acknowledgments}

Funding: None.

\section{Footnote}

Provenance and Peer Review: This article was commissioned by the editorial office, Annals of Translational Medicine. The article did not undergo external peer review.

Conflicts of Interest: Both authors have completed the ICMJE uniform disclosure form (available at http://dx.doi. org/10.21037/atm-20-4438). FL reports personal fees from Amgen, personal fees from Astellas, personal fees from Astra Zeneca, personal fees from Biontech, grants and personal fees from BMS, personal fees from Eli Lilly, personal fees from Elsevier, personal fees from Excerpta Medica, personal fees from Imedex, from Infomedica, grants and personal fees from Iomedico, personal fees from Medscape, from MedUpdate, personal fees from Merck Serono, personal fees from Merck Sharp Dohme, personal fees from Oncovis, personal fees from Promedicis, personal fees from Springer Nature, personal fees from StreamedUp!, from Zymeworks, personal fees from
Bayer, personal fees from Roche, outside the submitted work. The other author has no conflicts of interest to declare.

Ethical Statement: The authors are accountable for all aspects of the work in ensuring that questions related to the accuracy or integrity of any part of the work are appropriately investigated and resolved.

Open Access Statement: This is an Open Access article distributed in accordance with the Creative Commons Attribution-NonCommercial-NoDerivs 4.0 International License (CC BY-NC-ND 4.0), which permits the noncommercial replication and distribution of the article with the strict proviso that no changes or edits are made and the original work is properly cited (including links to both the formal publication through the relevant DOI and the license). See: https://creativecommons.org/licenses/by-nc-nd/4.0/.

\section{References}

1. Qin S, Ren Z, Meng Z, et al. Camrelizumab in patients with previously treated advanced hepatocellular carcinoma: 
a multicentre, open-label, parallel-group, randomised, phase 2 trial. Lancet Oncol 2020;21:571-80.

2. Chen X, Ma L, Wang X, et al. Reactive capillary hemangiomas: a novel dermatologic toxicity following anti-PD-1 treatment with SHR-1210. Cancer Biol Med 2019;16:173-81.

3. Huang J, Xu J, Chen Y, et al. Camrelizumab versus investigator's choice of chemotherapy as second-line therapy for advanced or metastatic oesophageal squamous cell carcinoma (ESCORT): a multicentre, randomised, open-label, phase 3 study. Lancet Oncol 2020;21:832-42.

4. Ringelhan M, Pfister D, O'Connor T, et al. The immunology of hepatocellular carcinoma. Nat Immunol 2018;19:222-32.

5. NCI Drug Dictionary [Internet]. National Cancer Institute. 2011. Available online: https://www.cancer.gov/ publications/dictionaries/cancer-drug

6. Markham A, Keam SJ. Camrelizumab: First Global Approval. Drugs 2019;79:1355-61.

7. Mo H, Huang J, Xu J, et al. Safety, anti-tumour activity, and pharmacokinetics of fixed-dose SHR-1210, an antiPD-1 antibody in advanced solid tumours: a doseescalation, phase 1 study. Br J Cancer 2018;119:538-45.

8. Zhu AX, Finn RS, Edeline J, et al. Pembrolizumab in patients with advanced hepatocellular carcinoma previously treated with sorafenib (KEYNOTE-224): a non-randomised, openlabel phase 2 trial. Lancet Oncol 2018;19:940-52.

9. Finn RS, Ryoo B-Y, Merle P, Kudo M, Bouattour M, Lim HY, et al. Pembrolizumab As Second-Line Therapy in Patients With Advanced Hepatocellular Carcinoma in KEYNOTE-240: A Randomized, Double-Blind, Phase III Trial. J Clin Oncol 2020;38:193-202.

10. El-Khoueiry AB, Sangro B, Yau T, et al. Nivolumab in patients with advanced hepatocellular carcinoma (CheckMate 040): an open-label, non-comparative, phase $1 / 2$ dose escalation and expansion trial. Lancet 2017;389:2492-502.

11. Abou-Alfa GK, Meyer T, Cheng AL, et al. Cabozantinib in Patients with Advanced and Progressing Hepatocellular Carcinoma. N Engl J Med 2018;379:54-63.

12. Bruix J, Qin S, Merle P, et al. Regorafenib for patients with hepatocellular carcinoma who progressed on sorafenib treatment (RESORCE): a randomised, double-blind, placebo-controlled, phase 3 trial. Lancet 2017;389:56-66.

13. Zhu AX, Kang YK, Yen CJ, et al. Ramucirumab after sorafenib in patients with advanced hepatocellular carcinoma and increased $\alpha$-fetoprotein concentrations (REACH-2): a randomised, double-blind, placebocontrolled, phase 3 trial. Lancet Oncol 2019;20:282-96.
14. Yau T, Park JW, Finn RS, et al. CheckMate 459: A randomized, multi-center phase III study of nivolumab (NIVO) vs sorafenib (SOR) as first-line (1L) treatment in patients (pts) with advanced hepatocellular carcinoma (aHCC). Ann Oncol 2019;30:v874-5.

15. Finn RS, Qin S, Ikeda M, et al. Atezolizumab plus Bevacizumab in Unresectable Hepatocellular Carcinoma. N Engl J Med 2020;382:1894-905.

16. Llovet JM, Ricci S, Mazzaferro V, et al. Sorafenib in Advanced Hepatocellular Carcinoma. N Engl J Med 2008;359:378-90.

17. Cheng AL, Kang YK, Chen Z, et al. Efficacy and safety of sorafenib in patients in the Asia-Pacific region with advanced hepatocellular carcinoma: a phase III randomised, double-blind, placebo-controlled trial. Lancet Oncol 2009;10:25-34.

18. Kudo M, Finn RS, Qin S, et al. Lenvatinib versus sorafenib in first-line treatment of patients with unresectable hepatocellular carcinoma: a randomised phase 3 noninferiority trial. Lancet 2018;391:1163-73.

19. Motz GT, Coukos G. The parallel lives of angiogenesis and immunosuppression: cancer and other tales. Nat Rev Immunol 2011;11:702-11.

20. Kudo M. Combination Cancer Immunotherapy with Molecular Targeted Agents/Anti-CTLA-4 Antibody for Hepatocellular Carcinoma. Liver Cancer 2019;8:1-11.

21. Voron T, Marcheteau E, Pernot S, et al. Control of the immune response by pro-angiogenic factors. Front Oncol 2014;4:70.

22. Pishvaian MJ, Lee MS, Ryoo BY, et al. Updated safety and clinical activity results from a phase Ib study of atezolizumab + bevacizumab in hepatocellular carcinoma (HCC). Ann Oncol 2018;29:viii718-9.

23. Llovet J, Shepard KV, Finn RS, et al. A phase Ib trial of lenvatinib (LEN) plus pembrolizumab (PEMBRO) in unresectable hepatocellular carcinoma (uHCC): Updated results. Ann Oncol 2019;30:v286-7.

24. Xu J, Zhang Y, Jia R, et al. Anti-PD-1 Antibody SHR-1210 Combined with Apatinib for Advanced Hepatocellular Carcinoma, Gastric, or Esophagogastric Junction Cancer: An Open-label, Dose Escalation and Expansion Study. Clin Cancer Res 2019;25:515-23.

Cite this article as: Catanese S, Lordick F. Anti-PD1 monotherapy in hepatocellular carcinoma: a step forward or already behind? Ann Transl Med 2020;8(24):1701. doi: 10.21037/ atm-20-4438 